\title{
Mycobacterial infection of a congenital bronchogenic cyst
}

\author{
WILIAM C HOUSER, GERALD J DORFF, DAVID Y ROSENZWEIG, AND \\ JOHN W AUSSEM
}

From the Department of Medicine, The Medical College of Wisconsin, and Department of Pathology, St Michael Hospital, Milwaukee, Wisconsin, USA

Bronchogenic cysts result from an abnormal budding or branching of the tracheobronchial tree. Approximately $30 \%$ of the cysts are located in the mediastinum and the remaining $70 \%$ in the lung. The cyst may be joined to the tracheobronchial tree by a fistula or by a fibrous band. Pulmonary bronchogenic cysts occur most frequently in the lower lobes, primarily on the right side. They are thin-walled, single or multiple, and lined with respiratory epithelium. The wall consists primarily of fibrous connective tissue and may contain mucous glands, hyaline cartilage, smooth muscle, nerve trunks, and elastic fibres. Bronchial communication with the cyst may be present. Intermittent patency of this communication allows accumulation of various combinations of air, fluid, blood, and purulent material within the cyst.

Approximately $15 \%$ of patients with bronchogenic cysts in reported series are asymptomatic. ${ }^{1-3}$ Respiratory symptoms in infants are primarily caused by expansion of the cyst with compression of adjacent tissue causing progressive respiratory distress and death. ${ }^{4}$ After infancy the symptoms are mainly related to complications including secondary infection, haemorrhage, perforation, and the occasional development of a malignant tumour. We report an asymptomatic patient with evidence of mycobacterial infection within a congenital bronchogenic cyst.

\section{Case report}

A 9-year-old white girl was admitted to a local hospital, because of recurrent epistaxis for the past two years. for cauterisation of bleeding sites on the nasal septum She had no symptoms attributable to her respiratory system and there was no known exposure to tuberculosis. Previous chest radiographs at ages $1,2,4$, and 6 years were normal. Past medical history included tonsillectomy and adenoidectomy three years earlier and three admissions to hospital for febrile seizures. There was no family history of tuberculosis or respiratory disease, and no other contacts with tuberculosis were known. She had not had BCG.

Physical examination revealed a healthy young girl. She was afebrile and there were no physical abnormalities. The haematocrit was $37 \%$, white blood cell count 4300 per $\mathrm{mm}^{3}$, with $39 \%$ polymorphs, $54 \%$ lymphocytes, $4 \%$ monocytes, and $3 \%$ eosinophils.

Address for reprint requests: Dr David Y Rosenzweig, Department of Pulmonary Medicine, The Medical College of Wisconsin, $8700 \mathrm{~W}$ Wisconsin Avenue, Milwaukee, WI53226, USA.
Admission chest radiographs (fig 1) showed a well defined $6 \mathrm{~cm}$ ovoid lesion in the posterior segment of the right lower lobe. It was thought wise to remove this and a right lower lobectomy was carried out. After ${ }_{\omega}^{+}$ completion of the procedure, the $6 \mathrm{~cm}$ cystic mass was dissected from the surrounding lung. No bronchialo communication was demonstrated. The lining of the cyst $\triangle$ was granular and trabeculated, with adherent shaggy, $\overrightarrow{-}$ friable light tan material. Using sterile technique, green purulent material was aspirated from the lesion for aerobic and anaerobic cultures. These cultures revealed $\overrightarrow{0}$ no growth. No specimen was submitted for myco-o bacterial culture. The histology of the resected specimen is shown in fig 2 . Kinyoun stain showed acid-fast bacilli within the areas of granulomatous inflammation.

The patient had an uneventful postoperative course. $\overline{\bar{O}}$ Her tuberculin skin test, $5 \mathrm{TU}$, was reactive tọ $12 \times 12 \mathrm{~mm}$ induration at 48 hours. Several sputa for尺 acid-fast bacilli stain and culture were negative. She was treated with rifampin $300 \mathrm{mg}$ per day for four monthsō and isoniazid $300 \mathrm{mg}$ with pyridoxine $30 \mathrm{mg}$ for two years. She remains asymptomatic; serial chest radio-? graphs for $\mathbf{3 0}$ months show stable postoperative changes.

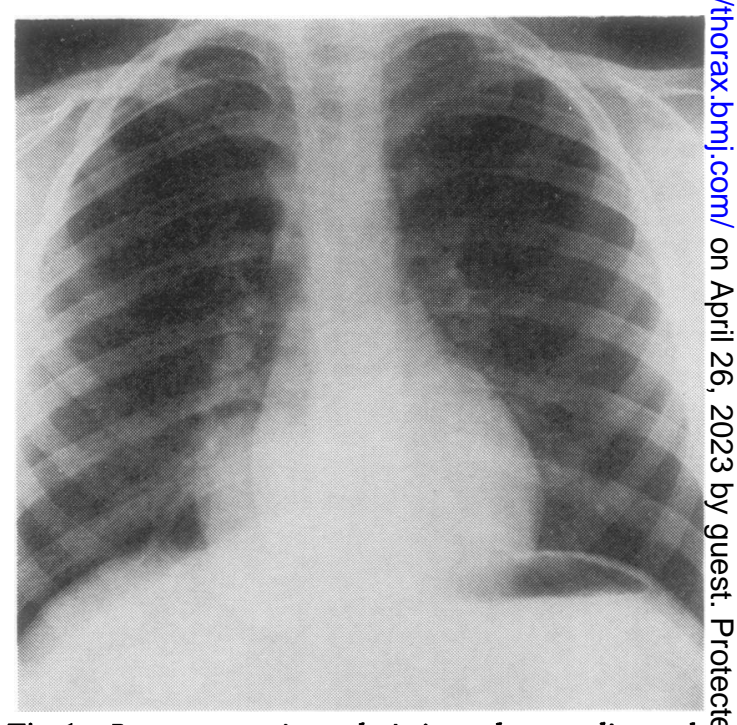

Fig 1 Postero-anterior admission chest radiograph, showing ovoid lesion posterolateral to the right cardiac border. 


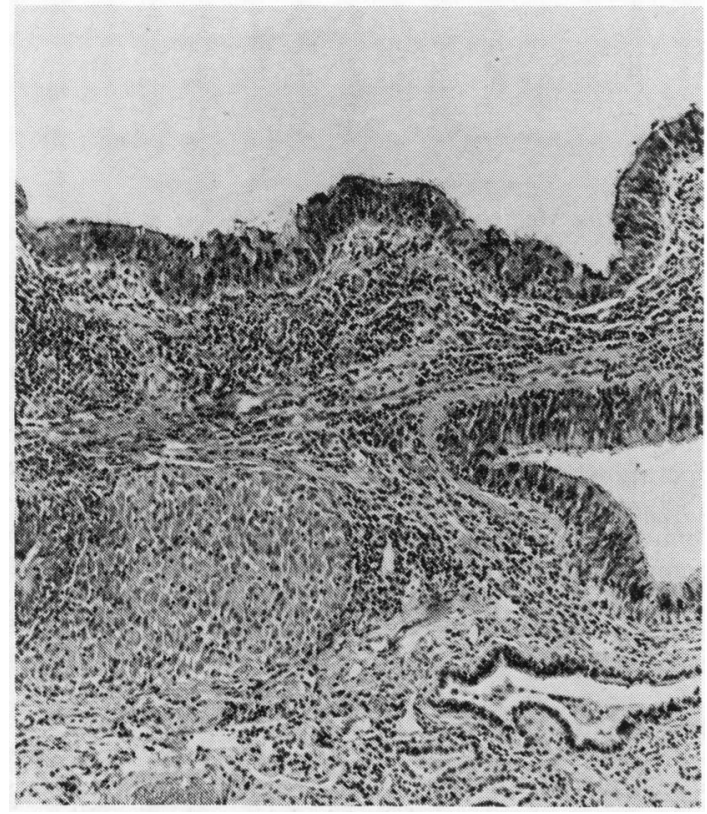

Fig 2 Photomicrograph of the lung cyst showing a ciliated columnar epithelial lining and extensive chronic inflammation consisting chiefly of lymphocytes and histiocytes immediately below the respiratory epithelium, and granuloma formation with numerous multinucleated giant cells and minimal caseation. Original magnification $\times 25$.

\section{Discussion}

The clinical manifestations associated with bronchogenic cysts in infants include respiratory distress, stridor, cyanosis, respiratory failure, and pneumothorax. Localised hyperinflation or collapse caused by bronchial compression by the cyst may occur. After infancy, the main symptoms are cough, chest pain, haemoptysis, dyspnoea, and fever. These symptoms are caused primarily by secondary bacterial infection; however, haemorrhage, superior vena caval obstruction, intracavitary aspergilloma, sarcoma, and carcinoma have been described. Bronchogenic cysts are also reported to be a frequent coexisting anomaly in patients with partial or complete absence of the pericardium. ${ }^{5}$ Because of the above complications and fatalities associated with bronchogenic cysts, the treatment of choice is surgical resection. Mycobacterial infection of bronchogenic cysts has not been described previously. The evidence for mycobacterial infection in the case presented includes the histology, the presence of acid-fast bacilli, and a positive tuberculin (5 TU) skin test in a 9-year-old girl who had not had BCG. Because the organism was not isolated, it is impossible to say if this was Mycobacterium tuberculosis or an atypical mycobacterial species. However, the relative rarity of atypical mycobacterial pulmonary infections in children would be against the latter. Since the patient is only 9 years old, this appears to be a manifestation of primary infection. The possible route of the mycobacterial infection in the cyst includes: (1) Direct deposition by ventilation. The cysts are characteristically poorly ventilated, suggesting that the possibility of cyst infection would be considerably less than infection of better ventilated areas of lung. However, if an organism gains access to the cyst, the defence mechanisms are likewise impaired, allowing persistence of the infection. (2) Direct extension from a pulmonary or lymph node focus. This is an attractive postulate because of the close proximity of the cyst to the perihilar area and the hilar lymphatics. (3) Haematogenous or lymphatic dissemination. Although this is a possibility, it does not appear to be a likely mechanism since no additional foci of dissemination were apparent.

Although our patient had had four previous chest radiographs, there was no evidence, even in retrospect, of a lesion. This is not unusual since Opsahl and Berman ${ }^{6}$ reported, from a review of the literature, that a previous abnormality was demonstrated by chest films in only 14 out of 25 symptomatic cases $(56 \%)$.

\section{References}

1 Rogers LF, Osmer JC. Bronchogenic cyst. Am J Roentgenol 1964; 91:273-83.

2 Moersch HJ, Clagett OT. Pulmonary cysts. J Thorac Surg 1947; 16:179-99.

3 Jones JC, Almond CH, Snyder HM, Meyer BW. Congenital pulmonary cysts in infants and children. Ann Thorac Surg 1967; 3:297-306.

4 Eraklis AJ, Griscom NT, McGovern JB. Bronchogenic cysts of the mediastinum in infancy. $N$ Engl J Med 1969; 281:1150-5.

5 Kwak DL, Stork WJ, Greenberg SD. Partial defect of the pericardium associated with a bronchogenic cyst. Radiology 1971; 101:287-8.

6 Opsahl T, Berman EJ. Bronchogenic mediastinal cysts in infants: case report and review of the literature. Pediatrics 1962; 30:372-7. 